\title{
DNA dynamics dictates p53 functional outcome
}

Jessy Safieh, Ariel Chazan, Pratik Vyas', Hanna Saleem, Yael Danin-Poleg, Dina Ron, and Tali E. Haran*

Faculty of Biology, Technion, Technion City, Haifa, Israel

${ }^{1}$ present address: Department of Biomolecular Sciences, Weizmann Institute of Science, Rehovot, Israel

* To whom correspondence should be addressed.

Email address: bitali@technion.ac.il

Phone: 972-4-8293767

Fax: 972-4-8225153 


\section{Abstract}

The tumor suppressor protein p53 is activated in response to cellular stress. An unresolved question in the field is how p53 is able to parse its myriad functions in a timely manner in accordance with the severity of the stress signal. Here we show across the genome that p53 target genes belonging to pathways acting early in the stress response have REs that are significantly more flexible than REs of genes involved in pathways that need to be more strictly regulated, or whose functional outcome occurs later in the response to stress. We validated these findings by several complementary experimental approaches, for six p53 REs belonging to pathways that operate at different times post p53 induction. Our results demonstrate that the flexibility of p53 REs contributes significantly to the temporal expression of p53 target genes and thereby to life versus death decisions in the p53 system. 
Gene regulation depends on the correct spatiotemporal arrangements of transcription factors and their specific DNA response elements (REs). It is now commonly accepted that recognition of DNA by regulatory proteins occurs not only by direct hydrogen bonds from the protein side chains to the DNA bases termed direct, or base, readout ${ }^{1}$, but is complemented by direct hydrogen bonds to the DNA backbone, termed indirect readout ${ }^{2}$, and by recognition of structural and dynamical (flexibility) properties of DNA REs, termed shape readout ${ }^{3}$.

The tumor suppressor protein p53 is part of a complex cellular network that is activated in response to various cellular stress signals. Activated p53 functions mainly as a transcription factor, regulating the expression of numerous genes, involved not only in various cellular pathways critical for preventing cancer, but also in pathways unrelated to cancer surveillance $e^{4,5}$. p53 is currently viewed more as the "guardian of homeostasis", rather than the "guardian of the genome" . It is now appreciated that p53 respond both to acute DNA damage and to more "routine" activities, such as energy metabolism ${ }^{7-10}$ and embryonic development ${ }^{11}$. In most of its activities p53 has both positive as well as negative outcomes, such as apoptosis and antiapoptosis $^{12-14}$.

A major question in the field is how p53 "chooses" the appropriate response to the acute or routine stress signals. Broadly, two alternative models were proposed ${ }^{15,16}$. The selective binding model focusses on inherent differences in p53 REs as a central driving force for discrimination between p53 target genes. The selective context model, suggests that $\mathrm{p} 53$ is not the crucial force behind target gene selectivity, but that discrimination is achieved by other events at the cellular and genomic level. Within the selective binding model, readout by base, indirect, and shape were all shown to contribute to the interaction of p53 with its target promoters ${ }^{17-25}$. In particular, we have previously demonstrated experimentally that DNA torsional (twist) flexibility changes significantly between p53 REs that differ in the uncontracted WW $(\mathrm{W}=\mathrm{A}, \mathrm{T})$ motif at the center of each $\mathrm{p} 53$ half-site ${ }^{18}$. Moreover, we have shown that when p53 levels are low, the torsional flexibility of p53 REs positively correlates with transactivation levels, whereas when p53 levels are highly induced, transactivation levels positively correlated with p53 binding affinity ${ }^{19}$.

Here, we hypothesize that across the genome p53-dependent genes with flexible REs belong to outcome categories in which there is a need for an early and immediate response upon stress. Rigid REs on the other hand, occur in functional categories where the response to stress is irreversible and likely to be more strictly regulated, or that it should occur at late stages in the response to stress. We show that this is indeed the case for all validated p53-dependent genes that are activated by direct binding of p53 to spacer-less REs ${ }^{25}$. Moreover, we prove this 
hypothesis using several complementary experimental approaches, in vitro and in cells, for six p53 REs belonging to genes in different outcome categories. Thus, the DNA torsional flexibility of p53 RE is important for singling out the time-wise appropriate target site among the numerous p53 dependent promoters. 


\section{Results}

\section{Classification of p53 REs by their functional categories yield distinct structural signature}

We have previously compiled a list of 250 p53 REs, validated for binding in cellular context and for p53-dependent gene expression ${ }^{25}$. These REs belong to 235 genes that are solely activating, do not contain spacer sequences between half-sites, and are found within the promoter or the first intron/exon region ${ }^{25}$. To classify these p53 genes, we looked for their specific function, the biological pathway (or process) initialized by them, and the functional outcome at the end of their biological pathway (Table S1). Functions were assigned to 219 of the 235 genes, some of which had more than one end outcome. For a meaningful statistical analysis, we added four more genes whose p53 REs reside in the second intron of the gene, thus, ending up with 223 genes and 240 p53 dependent REs. To assess the flexibility properties of these REs we used a parameter termed "deformability" that denotes the volume of conformational space within common energy contours ${ }^{26,27}$. We previously observed that this rigorous measure of helical flexibility, derived from crystal structures of protein-DNA complexes, has a strong and significant correlation with twist fluctuations (indicating twist flexibility) of p53 REs, measured using cyclization kinetics assays ${ }^{25}$. In analyzing the relation between deformability of p53 REs and their functional categories (Table S1), we took only one RE per gene for p53 REs clusters that contain more than two half-sites, assuming that p53 associates with all gene-specific REs when activating p53-dependent genes, yielding a single functional outcome. p53 clusters are known to confer additional binding affinity and increase transactivation levels from the nearby gene ${ }^{28,29}$. We chose to include only the most flexible fullsite RE of any given cluster, based on our previous study, where we showed that there is a weak but significant correlation between RE conservation (i.e., similarity to the consensus sequence) and deformability ${ }^{25}$. We therefore hypothesize here that the more conserved RE is the primary and dominant RE of a cluster of REs, and hence will be bound first $\mathrm{t}^{25}$. Three genes contained two partially overlapping REs consisting of three abutting half-sites, where the middle half-site is common to both REs (Table S1). In these cases we used the calculated deformability of the complete 30-bp RE, because of ambiguity in assigning the primary RE.

A boxplot of the deformability of p53 REs versus functional outcome of p53 activation, shows substantial differences in mean deformability values (denoted $\mathrm{V}(\mathrm{B})$ in units of ${ }^{03} \AA^{3}$ ) between functional categories (Fig. 1), when analyzing at least eight genes in each group, to minimize noise and scatter from small groups (Table 1 and S2). Results show that genes belonging to 533 positive-regulation category and genes belonging to survival and proliferation categories - angiogenesis, autophagy, cell-cycle arrest (CCA), development, DNA damage 
response activation (DDRA, i.e., just the activation phase of DDR that usually includes also CCA, repair and apoptosis), energy metabolism, and innate immunity, show higher than average deformability values (Table 1). Remarkably, the last four groups, those that are most needed for establishing a fast and timely response to cellular needs, have the highest deformability. On the other hand, genes belonging to death-related outcome (intrinsic apoptosis), to p53 negative regulation, and to a lesser extent, to cell adhesion (including both cell-cell and cell-ECM adhesion genes) and to cytoskeleton organization, have very low mean deformability values (Table 1).

The "survival" category (Fig. 1) comprise p53-dependent genes that inhibit death, such as anti-apoptosis, anti-necrotic and anti-pyroptosis genes, but nonetheless its deformability is slightly below average (Table 1). Note that the extrinsic (death receptor) apoptosis category, though it is death-related, has a slightly above average mean deformability (Fig.1 and Table 1). This is because the extrinsic-apoptosis pathway is initiated by signals originating from outside the cell, such as natural-killer lymphocytes, or cytotoxic-T lymphocytes, and thus are intimately connected with the innate- and adaptive-immune systems ${ }^{30}$. Interestingly, the REs of the autophagy category have deformability values that are either very high or very low (Table S2), as this group consists of two sub-groups that originate from two different connections. Autophagy genes having REs with high V(B) values $\left(2.59 \pm 0.07^{\circ 3} \AA^{3}\right)$ are linked to autophagy that comes together with the activation of innate-immunity genes, DNA damage response genes, or alternatively, connected with autophagy machinery genes (Table S2). Autophagy genes having REs with low $\mathrm{V}(\mathrm{B})$ values $\left(2.11 \pm 0.08^{\circ 3} \AA^{3}\right)$ are linked with autophagy that come together with apoptosis-related events (Table S2).

To assess the statistical significance of the differences in deformability between functional categories, we carried out one-way ANOVA on functional categories that contain at least eight genes each (total 178 genes, 18 categories, 223 functions). The significant results indicated that the groups are not sampled from populations with identical distributions $(F=$ 15.67, $P=<0.0001)$. A post-hoc test established that there was a significant difference in mean deformability between many functional groups (Table S3), the largest being between development and cell adhesion $(Z=4.998, P<0.0001)$. Of note to p53 functions as a tumor suppressor protein, are the differences between intrinsic apoptosis and CCA $(Z=-3.88, P=$ 0.013), intrinsic apoptosis and DDRA $(Z=-4.00, P=0.008)$, as well as between intrinsic and extrinsic apoptosis $(Z=-3.82, P=0.016)$. DNA repair, on the other hand, is one of the groups with high variations in deformability within the group, and thus its average does not differ 
significantly from intrinsic apoptosis $(Z=-3.24, P=0.11)$ or other categories. This is due mainly to the XPC RE, as further discussed below.

\section{Functional outcome categories have a unique sequence signature}

Sequence logos are a convenient way to depict base-pair preferences and information content of DNA binding sites ${ }^{31-33}$. We used sequence logos to assess DNA binding preferences of p53dependent functional groups. Here again, we analyzed the data by taking one RE per gene, except for the three sites containing three abutting half-sites, mentioned above, where we included both sites in the analysis (i.e., once where the common middle half-site is the 5' halfsite of RE1, and once where it is the 3' half-site of RE2). Fig. 2 shows representative patterns for selected functional groups (Fig. S1 shows all patterns), created by Weblogo ${ }^{32,33}$, displaying dramatic changes between them, in all positions, and especially relative to the degenerate pattern of all 250 REs together ${ }^{25}$. To quantitate the patterns observed in the sequence logos, we calculated Rseq for each pattern (Table S4). Rseq is defined as the total decrease in uncertainty (or in maximal entropy) per DNA binding site and is a measure for the information content of DNA binding sites ${ }^{31}$. When we calculate the ratio of Rseq of CWWG regions normalized by that of the whole site, within a specific outcome category, we divide the sequence logos to those in which the information content is high mainly in the two CWWG motifs, versus those in which it is high mainly in all other regions except CWWG (Fig. 2). The CWWG is the region with the uncontacted WW positions, and hence it is where shape-readout dominates ${ }^{18}$. In the present study these are the sites with particularly high information content, in one or both halfsites of the following categories: development ( $71 \%$ of total Rseq is in the CWWG regions), DDRA (74\%), energy metabolism (73\%), innate immunity (70\%), and to some extent CCA $(69 \%)$. These are the outcome categories where we observed high average deformability of their REs. This may mean that high information content (binding free-energy contributions of each base) in shape-readout positions comes together with high deformability (structural property of regions within the DNA double helix), and both occur in categories that are known to lead to immediate response upon stress. Indeed, Rseq of the two CWWG regions together is rank correlated to the deformability of full-sites REs $(\rho=0.53, P=0.02$ for 18 categories having at least eight genes each). Other sites, such as survival and membrane trafficking, seem to have moderately high information content in the two CWWG groups (67\% each), but in the case of survival, it is high also in non-CWWG regions, and thus survival has a high overall information content (16.3 \pm 0.8 bits). Membrane trafficking (and to some extent also CCA) may suffer from the lack of sufficient number of genes in this category (eight genes only each). The 
opposite pattern is observed in the sites with high information content in all positions except CWWG (i.e., the RRR and YYY regions), such as p53 negative regulation and extrinsic apoptosis ( $48 \%$ of the total Rseq is in CWWG regions). Nonetheless, these two categories have high overall information content $(16.3 \pm 1$ and $19.0 \pm 0.9$, respectively). In p53 negative regulation the $9^{\text {th }}$ and $12^{\text {th }}$ position are more conserved than those of the highly conserved $\mathrm{C}$ and $\mathrm{G}$ at the $4^{\text {th }}, 7^{\text {th }}$, and $17^{\text {th }}$ positions. This may mean that in these two categories the driving force for p53-DNA interaction is mainly the recognition of specific protein-DNA bases contacts, as these positions are known to be directly contacted by p53 in p53-DNA complexes, though some indirect readout is observed there as well ${ }^{17,20}$. Last, there are functional groups in which the information content is low at all positions, such as p53 positive regulation, which is highly variable at all but the $G$ at the $7^{\text {th }}$ position, which may mean that the functional outcome of this category has diverse origins, which are unrelated to DNA flexibility, or to specific protein-DNA contacts, and thus sequence conservation does not add up in the logo pattern. It should be noted that the correction for small sample size reduces the information content of the grouped sites ${ }^{31}$. Hence, the increased information content in individual categories is not an artifact of the smaller sample size relative to the 250 REs set.

\section{Experimental validation of the flexibility of six natural p53 REs by cyclization kinetics}

We used deformability to calculate the flexibility properties of p53-activating REs, above and before $^{25}$, because this parameter has a strong and significant correlation to experimental torsional flexibility of p53 sites, measured by cyclization kinetics ${ }^{25}$. Part of the data used to establish this correlation is the data on six well-known p53 REs studied here (Table 2). The chosen REs belong to genes that function at different cellular pathways, and act at different times post p53 induction. CCNG1, p21-5' and RRM2B functional outcome (CCA, DDRA, and DNA repair) occur earlier in the response to stress than PMAIP1 or TP53AIP1 (intrinsic apoptosis) and DDIT4 (negative p53 regulation). These REs span the entire range of deformability values of p53 REs (Table S1). The DDIT4 site, chosen for all experiments carried out in this study, is the less deformable site of the two p53 REs in the promoter of the DDIT4 gene, and was chosen because it is the least deformable p53 site in our entire p53 activating p53 dataset $^{25}$. As in our previous studies on p53 binding sites ${ }^{18,25,34}$, the only significant change in global-structural parameters of these sites is in the twist (torsional) flexibility of the DNA binding sites (Table 2). More importantly, CCNG1 is the most flexible site from the set of six sites chosen for experimental study, followed by RRM2B and p21-5'. At the bottom of the list are TP53AIP1 and DDIT4. This validates our hypothesis that p53 REs belonging to DDRA and 
CCA outcomes are more flexible than those belonging to intrinsic apoptosis and negative p53 regulation.

\section{Reporter gene assays as a function of p53 levels}

To validate our hypothesis in cellular context we carried out luciferase reporter gene assays in human non-small lung carcinoma cell line (H1299), ectopically expressing p53 along with reporter plasmids, each carrying a distinct RE. First, we ascertained that p53 ectopic expression is linearly correlated with the amount of transfected p53 expression plasmid pC53-SN3. This was done by transfecting cells with increasing amounts of p53 expression vector and a reporter construct harboring the p21-5' RE and immunoblotting the cell lysate (Fig. 3A). Densitometry analysis of the observed p53 band, normalized to the $\beta$-actin band confirmed the linear relationship between p53 protein level and pC53-SN3 amount $(\mathrm{R}=0.998$, Fig. 3B). We then carried out reporter-gene assays as a function of p53 levels for the six well-known p53 REs described above. We chose to analyze transactivation by reporter-gene assays $48 \mathrm{~h}$ after transfection, to enable observing a significant signal also in cells transfected with the lowest p53 plasmid amount (1 ng p53 plasmid). As can be observed from Fig. 4, in cells expressing low levels of p53, transactivation from flexible REs (RRM2B, p21-5' and CCNG1) was higher compared to transactivation from the more rigid REs (PMAIP1, TP53AIP1, and DDIT4). ANOVA analysis revealed that transactivation levels of the REs are significantly different from each other $\left(F=6.78, P=4 \times 10^{-3}, F=21.59, P=8.16 \times 10^{-7}\right.$ for cells transfected with $1 \mathrm{ng}$ or $2 \mathrm{ng}$ p53 plasmid, respectively). Post-hoc analysis revealed that in cells transfected with $1 \mathrm{ng}$ p53 plasmid, transactivation level from the p21-5' RE was significantly higher than that from PMAIP1, TP53AIP1 and DDIT4 REs. At 2 ng p53 plasmid, transactivation level from the RRM2B RE, or the CCNG1 RE, was significantly higher than that from PMAIP1, TP53AIP1 and DDIT4 REs, whereas transactivation level from p21-5' RE was significantly higher only to those from the TP53AIP1 and DDIT4 REs. At the highest amount of p53 (75 ng of pC53-SN3) all REs except DDIT4 exhibit similar levels of transactivation (Figure S4). The transactivation from cells transfected with $2 \mathrm{ng}$ p53 plasmid is strongly and significantly correlated with the experimental twist fluctuation of the six REs studied by cyclization kinetics $(\rho=0.986, P=3.1$ $X\left(0^{-4}\right)$, as we expected based on our earlier study ${ }^{19}$. Moreover, we calculated the difference between transactivation from the three most flexible REs and the three most rigid REs and found that the two groups differ significantly from each other at both p53 levels $(t=3.67, P=$ 0.021 and $t=3.88, P=0.018$ at $1 \mathrm{ng}$ and $2 \mathrm{ng}$ p53 plasmid, respectively). 


\section{Reporter gene assays as a function of time}

The higher transactivation level observed $48 \mathrm{~h}$ post transfection from flexible REs relative to rigid REs could be due to an earlier start of transactivation, or alternatively due to faster transactivation rates. To distinguish between these possibilities we examined transactivation levels, at $25 \mathrm{ng}$ p53 plasmid (to enable observation of a significant signal also at shorter times), at additional time points $(12,18$, and $24 \mathrm{~h}$ post transfection, Fig. 5). ANOVA analysis at $12 \mathrm{~h}$ post transfection revealed a small but significant difference between the transactivation level of the six REs $(F=3.16, P=0.03)$. A post-hoc analysis indicated that transactivation from CCNG1 is significantly different from DDIT4 (Tukey HSD $P=0.027$ ). At $18 \mathrm{~h}$ post infection, post-hoc analysis indicated that transactivation from RRM2B is significantly different from DDIT4 $(F=4.84, P=0.006$, Tukey HSD $P=0.0018)$. Only at $24 \mathrm{~h}$ post infection transactivation from most of the studied REs were significantly different from each other $(F=$ $35.75, P=9.75 \times 10^{-9}$ ), and similar to the differences at $48 \mathrm{~h}$. Spearman rank correlation between p53-dependent transactivation level from the six studied REs $24 \mathrm{~h}$ post transfection and their experimental twist fluctuations (indicating twist flexibility), established a positive strong and significant correlation between these two parameters $(\rho=0.96, P=0.0029)$. The difference between transactivation from the three most flexible REs and the three most rigid REs differ significantly from each other at all the time points, however, the most significant differences were observed at $24 \mathrm{~h}$ post transfection $(t=3.31, P=0.03)$.

\section{mRNA level from endogenic p53-dependent genes are correlated with p53 REs deformability and with their functional outcome}

In the gene-reporter system used in this study, p53 REs transactivation level is monitored from a transiently-transfected plasmid. To be able to mimic a more natural environment where transcription is affected by multiple other factors, we searched in the literature for data on mRNA levels of cellular genes in response to endogenous p53. Studies by the group of Lahav and colleagues have introduced the notion that different stress levels evoke different dynamic patterns of p53 expression ${ }^{35-40}$. Low stress level induces a pulsatile pattern of expression (the level of p53 expression exhibit a series of pulses with fixed amplitude and frequency), whereas more severe stress induces sustained pattern of expression (where only one continuous peak of p53 expression is observed). In the study by Purvis et al. ${ }^{40}$ the mRNA level of a large set of p53-dependent genes was measured by qPCR, every hour up to $24 \mathrm{~h}$ post-stress induction, for both pulsatile and sustained p53-expression pattern. To concur with our hypothesis, we took from this study only genes that p53 directly activates ${ }^{25}$. We also deleted genes that contain REs 
with long (> $9 \mathrm{bp}$ ) spacer sequences between the two half-sites, because p53 REs that harbor long spacer sequences can bind concurrently also in the hemi-specific mode of interaction ${ }^{34}$. Fig. 6 shows a strong and significant positive correlation between mRNA level $1 \mathrm{~h}$ post-stress induction and deformability of the REs, for both the pulsatile $\left(\rho=0.88, P=8.4 \times 10^{-5}\right)$ and the sustained mode of $\mathrm{p} 53$ expression $\left(\rho=0.85, P=2.6 \times 10^{-4}\right)$. No correlation was observed at times later than $1 \mathrm{~h}$. It is not surprising that the same correlation is observed for both pulsatile and sustained mode of p53 expression, since at $1 \mathrm{~h}$ they are still very similar. It is however, interesting to note that not only is there a correlation between mRNA level and deformability of the individual REs, but that they are grouped by their functional outcome. Highest deformability and mRNA level is observed for the two CCA genes (CDKN1A and GADD45A), then two genes that function both in autophagy as well as intrinsic apoptosis (BAX and PUMA), followed by genes that function only in intrinsic apoptosis (PMAIP1 and TP53AIP1), followed by three genes that function in p53 negative regulation (MDM2, PPM1D and XPC ${ }^{41}$ ). Moreover, XPC is mainly known to function in DNA repair ${ }^{42}$, which is a category with average flexibility REs. However, temporal coordination of the multistep repair process is crucial, and in it XPC is not the initial event ${ }^{42}$. The most rigid REs, and least amount of mRNA, are those for genes in which the two half-sites are interrupted by spacer sequences, DDB1 (1-bp spacer) ${ }^{43}$, and CASP6 (4-bp spacers) ${ }^{44}$, with CASP6 being worse than DDB1, in agreement with our previous observation on binding of p53 to REs with spacers ${ }^{34}$. Two additional REs appear in Fig. 4, BCL6 belonging to adaptive-immunity outcome, which we have not analyzed statistically because this group currently contain only four genes, and SESN1, belonging to oxidative-stress outcome (and mTor inhibition, Table S1). The average deformability of this category is $2.13 \pm 0.07^{\circ 3} \AA^{3}$, but SESN1 is an outlying REs within this category with $\mathrm{V}(\mathrm{B})$ below group average $\left(\mathrm{V}(\mathrm{B})=1.94^{\circ 3} \AA^{3}\right)$.

\section{Discussion}

Temporal expression of p53 target genes govern cell-fate decisions. An important question in the field is how p53 discriminates between the myriad p53-dependent target genes. Whereas the importance of differential binding affinities of p53 to its $\mathrm{REs}^{45}$, the dependence of cell-fate decisions on $\mathrm{p} 53$ levels $^{46-48}$, and on the elapsed time post p53 induction ${ }^{47}$ are widely acknowledged, the mechanistic basis for p53-dependent temporal gene expression is currently unknown. In our previous studies we showed experimentally that the torsional flexibility of the central CWWG motif is the major structural change between $553 \mathrm{REs}^{18}$, and that at high p53 levels transactivation from a given RE is determined by binding affinity, whereas at basal p53 
levels is it regulated by p53 binding kinetics, governed by DNA flexibility of the $\mathrm{RE}^{19}$. These mechanistic observations support the selective binding model, as a major contributor to targetgene selectivity in the p53 system, but by themselves do not explain the unique manner by which p53 can temporally coordinate its myriad target genes, to counteract an acute-stress signal, or a routine cellular demand. Here we show here that DNA flexibility is a strong classifier of p53 functional outcome. Genes that are part of outcome categories in which a response is needed immediately, time-wise, have REs that are capable of transactivating the genes at a faster rate and at low levels of p53, because they are flexible. Thus, our results obtained in vitro and in cells clearly indicate that the information for the selective binding of p53 to DNA is intrinsically encoded within the base sequence of the p53 REs. Hence, one should conclude that more than "p53 is smart"16, the DNA double helix where the REs reside is "smart"- the REs base sequences are information rich by virtue of their structural properties.

The dependence of the functional outcome of genes activation by p53 on RE flexibility can be illustrated by the cascade that begins with the detection of damage to DNA. It is known that initial stages of the response to DNA damage is an immediate event, preceding $\mathrm{CCA}^{49,50}$. Thus, the category we termed "DDR activation" (DDRA) should be an outcome category with very flexible REs, as shown in this study (Fig. 1, Table 2). The deformability of DDR REs is however not statistically different as a group from that of CCA-related REs, mainly because these two groups share few genes. Following CCA, p53 regulates the attempts at repairing the damage to DNA, unless the damage is too severe to be repaired, which depends on the accumulated level of DNA damage ${ }^{51}$. Intrinsic apoptosis should come after DNA-repair attempts, and only if the repair process failed ${ }^{52,53}$. This last step, being an irreversible step, is partly regulated by the high rigidity of the REs of apoptosis-related genes. Thus, the qualitative deformability series in acute stress - DDRA $>$ CCA $>$ DNA repair $>$ intrinsic apoptosis follows the order of events in the cells upon DNA damage. Autophagy and differentiation are additional functional outcomes that can be activated by p53, as an alternative mechanism to eliminate damaged cells ${ }^{54}$. Apoptosis-related autophagy (upper six REs in the autophagy category in Table S2) and differentiation genes have REs with deformability values in the range between those of DNA repair and intrinsic apoptosis. One should of course consider the spread of deformability values within each functional category, and likewise in the temporal order of events that takes place within each functional outcome group, as shown above for XPC. Additional example is RRM2B. RRM2B supplies nucleotides for the DNA-repair process ${ }^{55}$, thus it functions early in DNA repair. Indeed, it is the most deformable RE in the DNA-repair category (Table S2), is one of the most torsionally flexible sequence, as determined from the 
cyclization kinetics measurements, and within the whole category of DNA repair genes, it is an upper outlier (Fig. 1).

Another illustration of the relationship between flexibility of REs and functional outcome is depicted in genes that up- and down-regulate p53. Genes that positively regulate p53 levels and stability, at the start of a stress event, have REs with higher torsional flexibility relative to those that down-regulate p53 (Fig. 1, Table S2). Genes that down-regulate p53 possess more rigid REs, as these are transcribed towards the end of stress events when p53 levels should be decreased. Additional example, is the relationship between innate immunity a fast and early event, with genes harboring REs that are very flexible, versus adaptive immunity, which is not an immediate event, and its genes have relatively rigid REs (Table S2).

p53 sequence logo is known to be degenerate, as depicted in Fig. 2D, which is based on 250 validated activating REs that do not have spacers sequences between the half-sites ${ }^{25}$. Here we show that when we group p53 REs by their functional outcome, the pattern becomes more information rich. Degeneracy in consensus sequence may be critical for gene regulation, as it allows for variable temporal and p53 level-dependent response ${ }^{56}$. Indeed, we show here that p53 consensus-sequence pattern is a mixture of different functional outcomes that have different sequence/structure requirements. Some genes lead to early response and some to late ones, some genes are activated at basal p53 levels and some at highly induced levels. Putting them together obscure the fine details of the pattern.

\section{Online Methods}

\section{Cells, plasmids and reagents}

H1299 human non-small lung carcinoma cells (p53-null cells) were obtained from ATTC (CRL-5803). Cells were thawed and propagated in RPMI-1640 with 10\% Foetal Bovine Serum (FBS), 2 mM L-glutamine, and 1\% Pen-Strep solution (Biological Industries). All experiments were performed in this medium. Cells were maintained in a humidified incubator at $37{ }^{\circ} \mathrm{C}$ and $5 \% \mathrm{CO}_{2}$. Cells were routinely checked for mycoplasma infection and tested negative.

The reporter gene vectors pCLuc Mini- TK 2, encoding Cypridina secreted luciferase under the control of minimal promoter, and pCMV-GLuc, encoding constitutively expressed Gaussia secreted luciferase, were purchased from New England Biolabs. pC53-SN3 construct (where p53 expression is driven by the CMV promoter) was used for wild-type p53 expression in H1299 cells. p53 expression plasmid was kindly provided by Varda Rotter, Weizmann Institute of Science, Rehovot, Israel. 


\section{Generation of reporter plasmids containing p53 REs}

All DNA sequences were synthesized by Sigma Genosys (Israel) and purified by standard desalting. The sequences corresponding to p53 REs were incorporated in pCluc Mini-TK plasmids using site-directed mutagenesis with KAPA HiFi ${ }^{\mathrm{TM}}$ DNA Polymerase kit (Gamidor Diagnostics), and transformed into competent DH5 $\alpha$ cells, according to manufacturer recommendations. DNA Primers used for incorporation are found in Table S5. Overexpressed plasmids were extracted using QIAprep® Spin Miniprep Kit (Qiagen), according to manufacturer's protocol. The identity of incorporated sequence were verified using Sanger sequencing.

\section{Reporter gene assays}

H1299 cells were seeded in 24-well plates at a density of 5X10 4 cells/well and incubated for 24 h. Then cells were co-transfected with $800 \mathrm{ng}$ of either pCLuc Mini-TK 2 harboring p53 RE or a corresponding empty vector along with $50 \mathrm{ng}$ of pCMV-GLuc and 0 to $75 \mathrm{ng}(0,1,2,5,10$, 25, $75 \mathrm{ng}$ ) of p53 expression vector (pC53-SN3). Total DNA amount was adjusted using pcDNA 3.1 plasmid. Transfection was performed with jetPEI (Polyplus) reagent according to the manufacturer's recommendation. Transfection efficiency was monitored by transfecting separate wells with $0.1 \mu \mathrm{g}$ eGFP along with Gluc and pcDNA plasmids. An aliquot of growth medium $(20 \mu \mathrm{l})$ was taken at various time points post transfection and luminescence was measured using Pierce ${ }^{\mathrm{TM}}$ Cypridina Luciferase Flash Assay Kit and Pierce ${ }^{\mathrm{TM}}$ Gaussia Luciferase Flash Assay Kit (both from Thermo Fisher) according to the manufacturer's protocol. Signal was detected by CLARIOstar 96 well plate reader (BMG LABTECH). Four to six independent experiments were carried out, each containing two technical replicates.

\section{Analysis of transactivation from p53 REs}

To correct for transfection variability, all results from Cypridina Luciferase luminescence were normalized to Gaussia Luciferase luminescence. Next, the results were normalized in two steps. First, the results obtained with pCLuc Mini-TK 2 harboring p53RE $\left(\mathrm{Cluc}^{+\mathrm{RE}}\right)$ were divided by the results obtained with the corresponding empty vector $\left(\mathrm{Cluc}^{-\mathrm{RE}}\right)$, Second, the results obtained with p53 (1-75ng of pC53-SN3) were divided by the results obtained without p53 (0 ng of pC53-SN3). Fold increase in transactivation is thus given by the equation:

Fold increase in transactivation $=\left(\mathrm{Cluc}^{+\mathrm{RE}} / \mathrm{Cluc}^{-\mathrm{RE}}\right)^{+\mathrm{p} 53} /\left(\mathrm{Cluc}^{+\mathrm{RE}} / \mathrm{Cluc}-\mathrm{RE}\right)^{-\mathrm{p} 53}$ 


\section{p53 protein quantitation and detection}

Cells were lysed in RIPA buffer ( $150 \mathrm{mM} \mathrm{NaCl}, 1 \% \mathrm{NP}-40,0.5 \% \mathrm{Na}$ deoxychlate, $0.1 \%$ SDS, $25 \mathrm{mM}$ Tris-Cl, pH 7.5) containing 1/100 (Vol/Vol) Protease inhibitor cocktail (Sigma). Equal amount of protein from the different samples were resolved on $10 \%$ SDS PAGE, and transferred to PVDF membrane. The blots were subsequently incubated for $24 \mathrm{~h}$ at $4^{\circ} \mathrm{C}$ with primary antibodies against p53 (DO-7, Cell Signaling) and $\beta$ actin (13E5, Cell Signaling). Membranes were then reacted with a secondary antibody (Anti-mouse IgG, and Anti-rabbit IgG) conjugated to Horseradish Peroxidase (HRP), developed using Western Bright ${ }^{\mathrm{TM}}$ enhanced chemiluminescence reagent (Advansta), air-dried, and exposed using Fusion Pulse (Vilber) detection system. Blots were quantitated using Evolution-Capt software that came with the detection system.

\section{Cyclization kinetics and simulation of cyclization data}

DNA constructs for cyclization kinetics were synthesized using the top library/bottom test sequence PCR scheme, described previously ${ }^{18,25,34,57}$, except that here we used as test sequences DNA sequences containing the full 20-bp long p53 sites plus 5-bp flanking sequences on either side, specific for each natural p53 site (see Table S6 for sequences), because we have previously shown that sequences flanking p53 REs modulate p53 binding and transactivation ${ }^{25}$. However, the global structural characteristics of the sites, (Table 1, Fig. S2), are those of the central 20bp p53 sites without flanks. The DNA test sequences for cyclization experiments (bottom PCR templates) were synthesized by Sigma Genosys (Israel), whereas the library DNA sequences (top PCR templates) and the fluorescein- and tetramethylrhodamine (TAMRA)-labeled oligonucleotide primers were synthesized by the Keck foundation Laboratory at Yale University. Cyclization kinetics measurements were carried out as described previously ${ }^{18,25,34}$. PCR reactions $(50 \mu \mathrm{l})$ contained $1 \times$ PrimeSTAR buffer, $0.2 \mathrm{mM}$ dNTP mix, $0.3 \mu \mathrm{M}$ of each of the primers, $100 \mathrm{nM}$ of the top and bottom templates and 0.04 units of PrimeSTAR DNA polymerase (Takara, Japan). Ligase concentration was varied as a function of phasing length $(0.08 \mathrm{U} / \mu \mathrm{l}$ for the in-phase $156 \mathrm{~L} 14$ and $156 \mathrm{~L} 16$ constructs and $1.0 \mathrm{U} / \mu \mathrm{l}$ for all other phasing constructs) and total length (from $0.08 \mathrm{U} / \mu \mathrm{l}$ for the 157 constructs, to $0.8 \mathrm{U} / \mu \mathrm{l}$ for the $154-155$ and $158-160$ constructs and $2.5 \mathrm{U} / \mu 1$ for the $150-153$ constructs). We derived quantitative data on the conformational properties of the test sequences by simulating the cyclization data as previously described ${ }^{18,25,34,58}$. 


\section{Functional annotation of p53 genes and grouping into pathways}

We previously assembled a set of 250 p53 REs without spacers that contain sites validated for binding in cellular context and for p53-dependent gene expression ${ }^{25}$ that belong to 235 genes (some genes contain multiple REs). We located each gene in the Genecards suite (https://www.genecards.org/) by their Entrez ID, gene symbol and gene name. We then looked for existing functional annotations in UniProtKB/Swiss-Prot (https://www.uniprot.org), extracted from the Gene Ontology (GO) project, derived from the 'Biological process' and 'Molecular function' categories, but only when a relevant publication was available. We then looked for more recent publications for each gene product. All our annotations are thus supported by individual publications, given in Table S1 by their Pubmed ID (PMID) codes. For each gene we looked for its specific function, the biological pathway or process involved, and the functional outcome at the end of the biological cascade initiated by the specific gene.

\section{Information content analysis}

Information content per DNA binding site (Rseq) was calculated separately for each p53 outcome category, using the method of Schneider et al. ${ }^{31}$, with the correction for small sample size (i.e., small number of DNA sequences), suggested in this study. Consensus sequence patterns ("logos") were created using Weblogo 3.7.4., based on ${ }^{32,33}$. The correction for small sample size is needed because one cannot easily compare the information content of groups of sequences differing in their number, especially when only limited sequence data exists, because limited data results in underestimation of the entropy per DNA site ${ }^{31}$. However, the correction for errors resulting from small sample size is known to minimize this bias ${ }^{31}$ and is also the default option in Weblogo.

\section{Other computational and statistical tests}

Testing the strength of relationships between variables was carried out by Spearman's rank correlation as a non-parametric measure of correlation. In these tests we used ties whenever the values were within \pm SEM of each other. In testing for statistically significant differences between functional groups grouped by their main p53 dependent functional outcome, as well as for differences in transactivation level of the studied REs, we used one-way analysis of variance (ANOVA). We used the Steel-Dwass method as a post-hoc test, to determine the groups that are significantly differed from each other by their deformability, because this test is adjusted for multiple comparisons, and the Tukey HSD $(\alpha=0.05)$ as a post-hoc test, for the differences between transactivation levels. All analyzes were conducted using JMP $®$, Version 

made available under aCC-BY-NC-ND 4.0 International license.

15 (SAS Institute Inc., Cary, NC, 2019). The values for the deformability (V(B) in units of ${ }^{03} \AA^{3}$ ) of each DNA base-pair step were calculated as previously described ${ }^{25}$, using the values of $^{27}$. 


\section{References}

1. Seeman, N.C., Rosenberg, J.M. \& Rich, A. Sequence-specific recognition of double helical nucleic acids by proteins. Proc. Natl. Acad. Sci. USA 73, 804-808 (1976).

2. Otwinowski, Z. et al. Crystal structure of trp repressor/operator complex at atomic resolution [published erratum appears in Nature 1988 Oct 27;335(6193):837]. Nature 335, 321-329 (1988).

3. Rohs, R. et al. Origins of specificity in protein-DNA recognition. Annu. Rev. Biochem. 79, 233-269 (2010).

4. Vousden, K.H. \& Prives, C. Blinded by the Light: The Growing Complexity of p53. Cell 137, 413-431 (2009).

5. $\quad$ Kastenhuber, E.R. \& Lowe, S.W. Putting p53 in Context. Cell 170, 1062-1078 (2017).

6. Aylon, Y. \& Oren, M. The Paradox of p53: What, How, and Why? Cold Spring Harb. Perspect. Med. 6, a026328 (2016).

7. Gottlieb, E. \& Vousden, K.H. p53 regulation of metabolic pathways. Cold Spring Harb. Perspect. Biol. 2, a001040 (2010).

8. Liang, Y., Liu, J. \& Feng, Z. The regulation of cellular metabolism by tumor suppressor p53. Cell Biosci. 3, 9 (2013).

9. Lacroix, M., Riscal, R., Arena, G., Linares, L.K. \& Le Cam, L. Metabolic functions of the tumor suppressor p53: Implications in normal physiology, metabolic disorders, and cancer. Mol. Metab. 33, 2-22 (2020).

10. Lahalle, A. et al. The p53 Pathway and Metabolism: The Tree That Hides the Forest. Cancers (Basel) 13, 133 (2021).

11. Koifman, G., Aloni-Grinstein, R. \& Rotter, V. p53 balances between tissue hierarchy and anarchy. J. Mol. Cell. Biol. 11, 553-563 (2019).

12. Portt, L., Norman, G., Clapp, C., Greenwood, M. \& Greenwood, M.T. Anti-apoptosis and cell survival: a review. Biochim. Biophys. Acta 1813, 238-259 (2011).

13. Sheikh, M.S. et al. The antiapoptotic decoy receptor TRID/TRAIL-R3 is a p53regulated DNA damage-inducible gene that is overexpressed in primary tumors of the gastrointestinal tract. Oncogene 18, 4153-4159 (1999).

14. Pan, G., Ni, J., Yu, G., Wei, Y.F. \& Dixit, V.M. TRUNDD, a new member of the TRAIL receptor family that antagonizes TRAIL signalling. FEBS Lett. 424, 41-45 (1998).

15. Espinosa, J.M. Mechanisms of regulatory diversity within the p53 transcriptional network. Oncogene 27, 4013-4023 (2008).

16. Vousden, K.H. p53: death star. Cell 103, 691-694 (2000).

17. Kitayner, M. et al. Structural basis of DNA recognition by p53 tetramers. Mol. Cell 22 741-753. (2006).

18. Beno, I., Rosenthal, K., Levitine, M., Shaulov, L. \& Haran, T.E. Sequence-dependent cooperative binding of p53 to DNA targets and its relationship to the structural properties of the DNA targets. Nucleic Acids Res. 39, 1919-1932 (2011).

19. Jordan, J.J. et al. Low-level p53 expression changes transactivation rules and reveals superactivating sequences. Proc. Natl. Acad. Sci. USA 109, 14387-14392 (2012).

20. Kitayner, M. et al. Diversity in DNA recognition by p53 revealed by crystal structures with Hoogsteen base pairs. Nat. Struct. Mol. Biol. 17, 423-429 (2010).

21. Chen, Y., Dey, R. \& Chen, L. Crystal structure of the p53 core domain bound to a full consensus site as a self-assembled tetramer. Structure 18, 246-256 (2010).

22. Chen, Y. et al. Structure of p53 binding to the BAX response element reveals DNA unwinding and compression to accommodate base-pair insertion. Nucleic Acids Res. 41, 8368-8376 (2013). 
23. Zhang, X. et al. Conformations of p53 Response Elements in Solution Deduced Using Site-Directed Spin Labeling and Monte Carlo Sampling. Nucleic Acids Res. 42, 27892797 (2014).

24. Golovenko, D. et al. New Insights into the Role of DNA Shape on Its Recognition by p53 Proteins. Structure 26, 1-14 (2018).

25. Senitzki, A. et al. The complex architecture of p53 binding sites. Nucleic Acids Res. 49, 1364-1382 (2021).

26. Olson, W.K., Gorin, A.A., Lu, X.J., Hock, L.M. \& Zhurkin, V.B. DNA sequencedependent deformability deduced from protein-DNA crystal complexes. Proc. Natl. Acad. Sci. USA 95, 11163-11168 (1998).

27. Balasubramanian, S., Xu, F. \& Olson, W.K. DNA sequence-directed organization of chromatin: structure-based computational analysis of nucleosome-binding sequences. Biophys. J. 96, 2245-2260 (2009).

28. Bourdon, J.C. et al. Further characterisation of the p53 responsive element-identification of new candidate genes for trans-activation by p53. Oncogene 14, 85-94. (1997).

29. Riley, T., Sontag, E., Chen, P. \& Levine, A. Transcriptional control of human p53regulated genes. Nat. Rev. Mol. Cell Biol. 9, 402-412 (2008).

30. Ricci, M.S. \& El-Deiry, W.S. The Extrinsic Pathway of Apoptosis. in Apoptosis, Senescence, and Cancer. Cancer Drug Discovery and Development series (eds. Gewirtz, D.A., Holt, S.E. \& Grant, S.) (Humana Press, 2007).

31. Schneider, T.D., Stormo, G.D., Gold, L. \& Ehrenfeucht, A. Information content of binding sites on nucleotide sequences. J. Mol. Biol. 188, 415-31 (1986).

32. Schneider, T.D. \& Stephens, R.M. Sequence logos: a new way to display consensus sequences. Nucleic Acids Res. 18, 6097-6100 (1990).

33. Crooks, G.E., Hon, G., Chandonia, J.M. \& Brenner, S.E. WebLogo: a sequence logo generator. Genome Res. 14, 1188-1190 (2004).

34. Vyas, P. et al. Diverse p53/DNA binding modes expand the repertoire of p53 response elements. Proc. Natl. Acad. Sci. USA 114, 10624-10629 (2017).

35. Hafner, A. et al. p53 pulses lead to distinct patterns of gene expression albeit similar DNA-binding dynamics. Nat. Struct. Mo.l Biol. 24, 840-847 (2017).

36. Batchelor, E., Loewer, A. \& Lahav, G. The ups and downs of p53: understanding protein dynamics in single cells. Nat. Rev. Cancer 9, 371-377 (2009).

37. Batchelor, E., Mock, C.S., Bhan, I., Loewer, A. \& Lahav, G. Recurrent initiation: a mechanism for triggering p53 pulses in response to DNA damage. Mol. Cell 30, 277289 (2008).

38. Loewer, A., Batchelor, E., Gaglia, G. \& Lahav, G. Basal dynamics of p53 reveal transcriptionally attenuated pulses in cycling cells. Cell 142, 89-100 (2010).

39. Batchelor, E., Loewer, A., Mock, C. \& Lahav, G. Stimulus-dependent dynamics of p53 in single cells. Mol. Syst. Biol. 7, 488 (2011).

40. Purvis, J.E. et al. p53 dynamics control cell fate. Science 336, 1440-1444 (2012).

41. Krzeszinski, J.Y. et al. XPC promotes MDM2-mediated degradation of the p53 tumor suppressor. Mol. Biol. Cell 25, 213-221 (2014).

42. Ribeiro-Silva, C. et al. Ubiquitin and TFIIH-stimulated DDB2 dissociation drives DNA damage handover in nucleotide excision repair. Nat. Commun. 11, 4868 (2020).

43. Nguyen, T.T. et al. Revealing a human p53 universe. Nucleic Acids Res. 46, 8153-8167 (2018).

44. Yang, C. et al. Transcriptional activation of caspase- 6 and -7 genes by cisplatin-induced p53 and its functional significance in cisplatin nephrotoxicity. Cell Death Differ. 15, 530-544 (2008). 
45. Weinberg, R.L., Veprintsev, D.B., Bycroft, M. \& Fersht, A.R. Comparative binding of p53 to its promoter and DNA recognition elements. J. Mol. Biol. 348, 589-596. (2005).

46. Chen, X., Ko, L.J., Jayaraman, L. \& Prives, C. p53 levels, functional domains, and DNA damage determine the extent of the apoptotic response of tumor cells. Genes Dev $\mathbf{1 0}$, 2438-2451 (1996).

47. Zhao, R. et al. Analysis of p53-regulated gene expression patterns using oligonucleotide arrays. Genes Dev. 14, 981-993. (2000).

48. Kracikova, M., Akiri, G., George, A., Sachidanandam, R. \& Aaronson, S.A. A threshold mechanism mediates p53 cell fate decision between growth arrest and apoptosis. Cell Death Differ. 20, 576-588 (2013).

49. Niida, H. \& Nakanishi, M. DNA damage checkpoints in mammals. Mutagenesis 21, 39 (2006).

50. Surova, O. \& Zhivotovsky, B. Various modes of cell death induced by DNA damage. Oncogene 32, 3789-3797 (2013).

51. Offer, H. et al. The onset of p53-dependent DNA repair or apoptosis is determined by the level of accumulated damaged DNA. Carcinogenesis 23, 1025-1032 (2002).

52. Elmore, S. Apoptosis: a review of programmed cell death. Toxicol. Pathol. 35, 495-516 (2007).

53. Nowsheen, S. \& Yang, E.S. The intersection between DNA damage response and cell death pathways. Exp. Oncol. 34, 243-254 (2012).

54. Helton, E.S. \& Chen, X. p53 modulation of the DNA damage response. J. Cell. Biochem. 100, 883-896 (2007).

55. Yamaguchi, T. et al. p53R2-dependent pathway for DNA synthesis in a p53-regulated cell cycle checkpoint. Cancer Res. 61, 8256-8262 (2001).

56. Hoh, J. et al. The p53MH algorithm and its application in detecting p53-responsive genes. Proc. Natl. Acad. Sci. USA 99, 8467-8472. (2002).

57. Zhang, Y. \& Crothers, D.M. High-throughput approach for detection of DNA bending and flexibility based on cyclization. Proc. Natl. Acad. Sci. USA 100, 3161-3166. (2003).

58. Zhang, Y. \& Crothers, D.M. Statistical mechanics of sequence-dependent circular DNA and its application for DNA cyclization. Biophys. J. 84, 136-153. (2003).

59. Zhang, Y., Xi, Z., Hegde, R.S., Shakked, Z. \& Crothers, D.M. Predicting indirect readout effects in protein-DNA interactions. Proc. Natl. Acad. Sci. USA 101, 8337-8341 (2004). 


\section{Figure legends:}

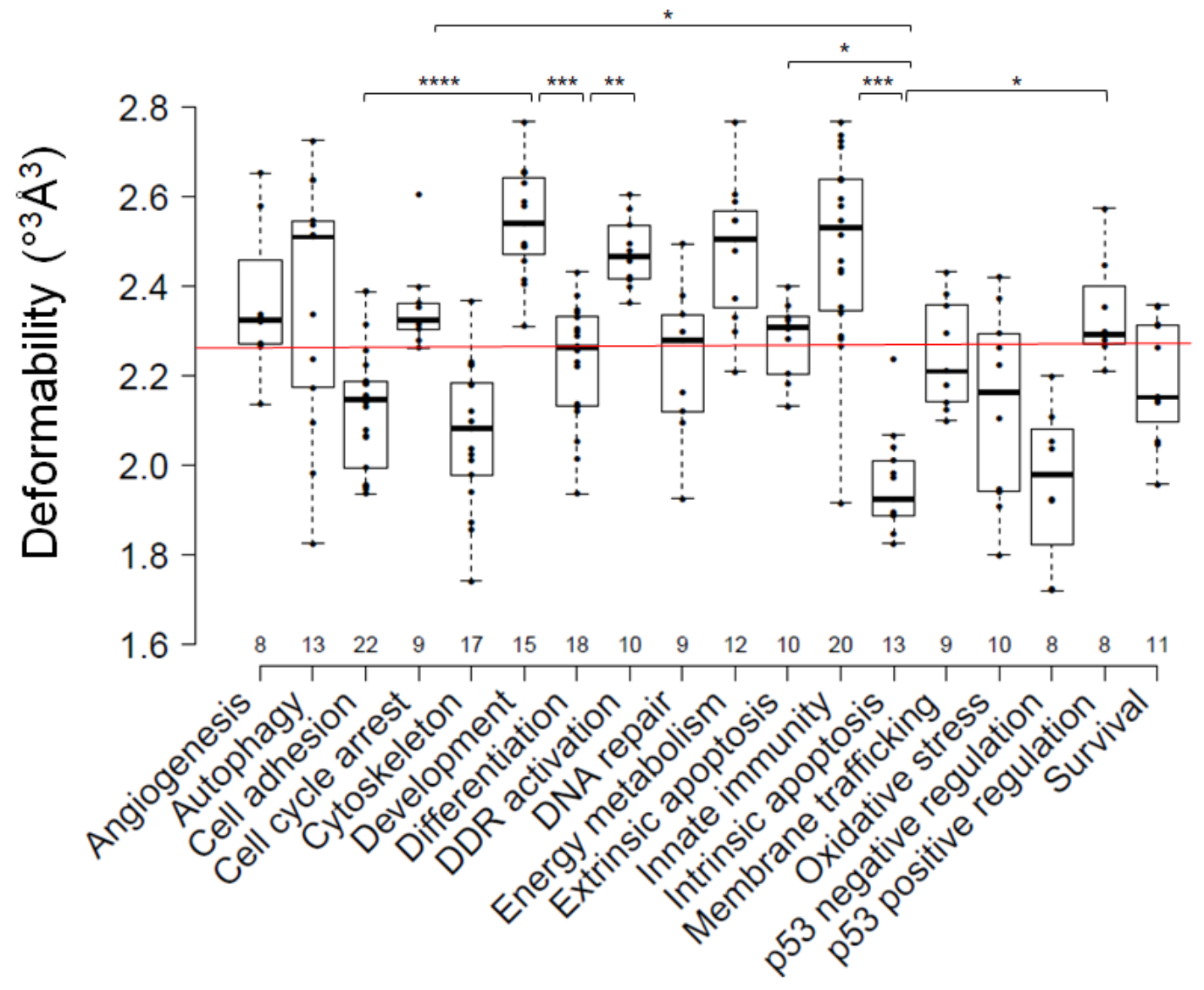

Fig. 1: p53 REs belonging to different functional categories are clustered by their deformability. Shown are functional categories with at least 8 genes each. Center lines show the medians; box limits indicate the 25th and 75th percentiles; whiskers extend 1.5 times the interquartile range from the 25 th and 75 th percentiles. All data points are shown as dots. The outlier point above CCA is BTG2, and that above intrinsic apoptosis is BAX. The red line marks the average deformability of the entire set used in the analysis. Numbers above the $\mathrm{x}$ axis mark the number of REs in each functional category. $\mathrm{P}$ values are marked as follows: $\mathrm{P}<0.03 *, \mathrm{P}<0.01 * *, \mathrm{P}<0.001 * * *, \mathrm{P}<0.0001 * * * *$. Only representative differences are marked by stars, to avoid clattering the pattern. For full list of significant differences see Table S3. 
a High Req values at CWWG positions

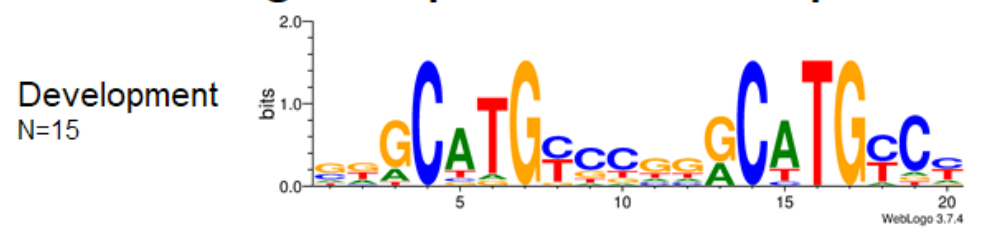

DNA

damage response $\mathrm{N}=10$

Energy metabolism $\mathrm{N}=14$
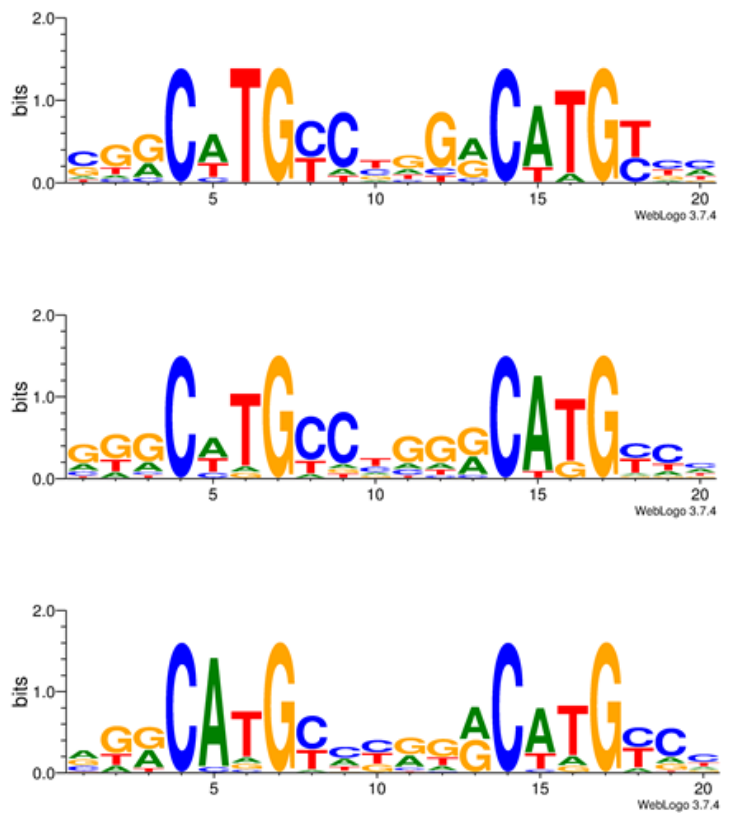

b High Req values at RRR/YYY positions

Extrinsic apoptosis $\mathrm{N}=10$

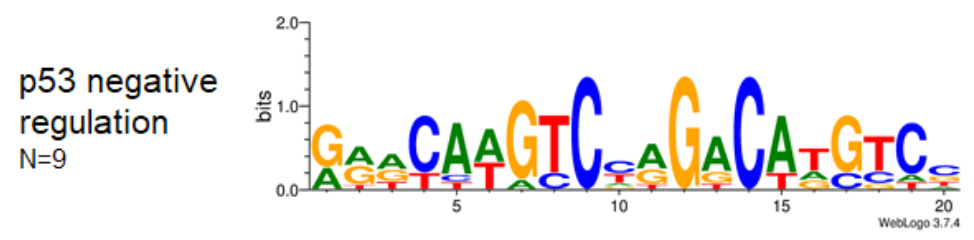

c

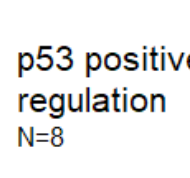

d

Low Req values in all positions

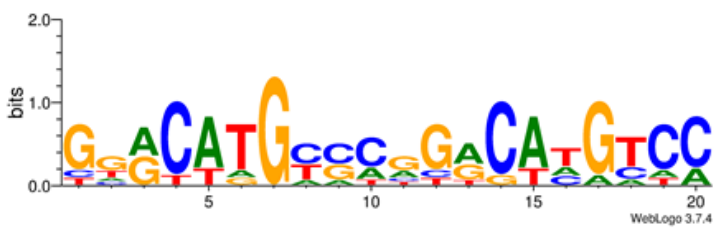

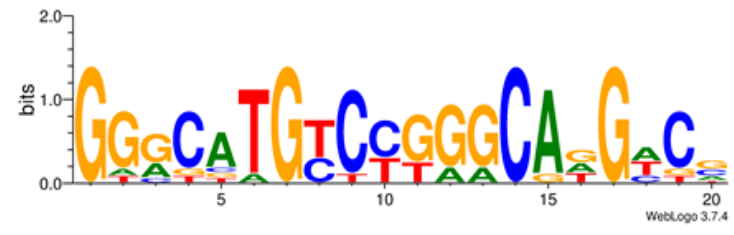

Consensus of 250 p53 REs (ref. 25)

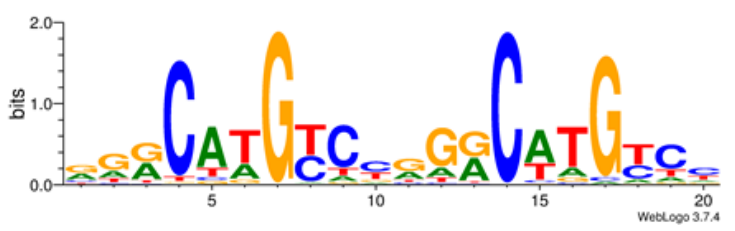

Fig. 2: Consensus p53-binding motifs by p53-dependent functional outcome categories. (A) Functional outcome categories with high Req values mainly at the two CWWG motifs. (B) Functional outcome categories with high Req values mainly at the RRR/YYY motifs. (C) Lowest Req values category throughout the entire site. (D) Consensus motif of the entire set of 250 activating p53 REs without spacers. Sequence motifs were generated by weblogs $31-33$

22 


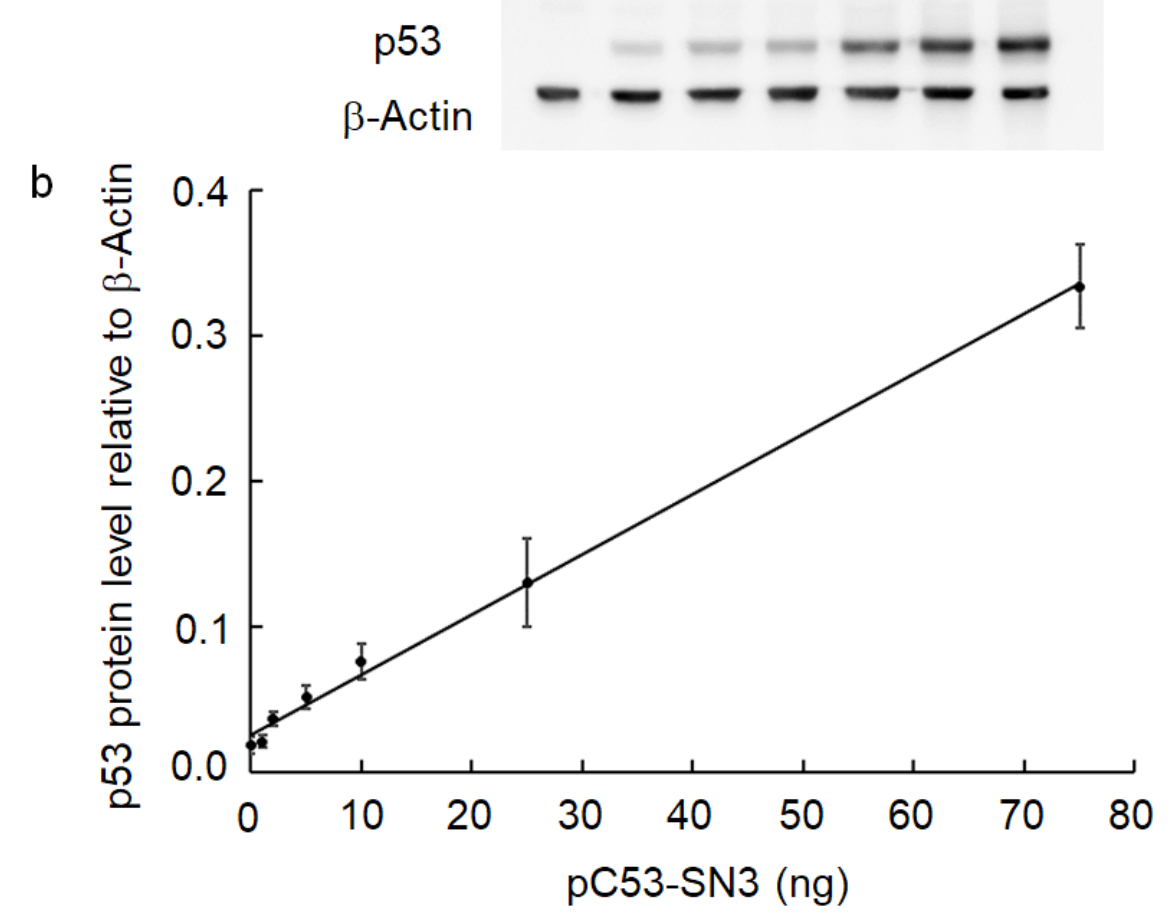

Fig. 3: Validation of ectopic p53 expression level in H1299 cells. (A) H1299 cells were transfected with increasing amount of p53 expressing vector, and a reporter construct harboring the p21-5' RE. After $48 \mathrm{~h}, 10 \mu \mathrm{g}$ of whole cells lysate was analyzed by immunoblotting with antibody against $\mathrm{p} 53$. $\beta$-Actin serve as control. (B) Correlation between $\mathrm{pC53-SN3}$ plasmid amount at the transfection against $\mathrm{p} 53$ protein level relative to $\beta$-actin, derived from (A). p53 protein level was estimated by normalizing the band intensity of p53 protein to the band intensity of $\beta$-Actin from the same sample. There is a linear relationship between the amounts of p53 expressing plasmid used in the transfection and the observed p53 protein levels $(\mathrm{R}=0.998)$. The results are averages of four independent experiments. 
a

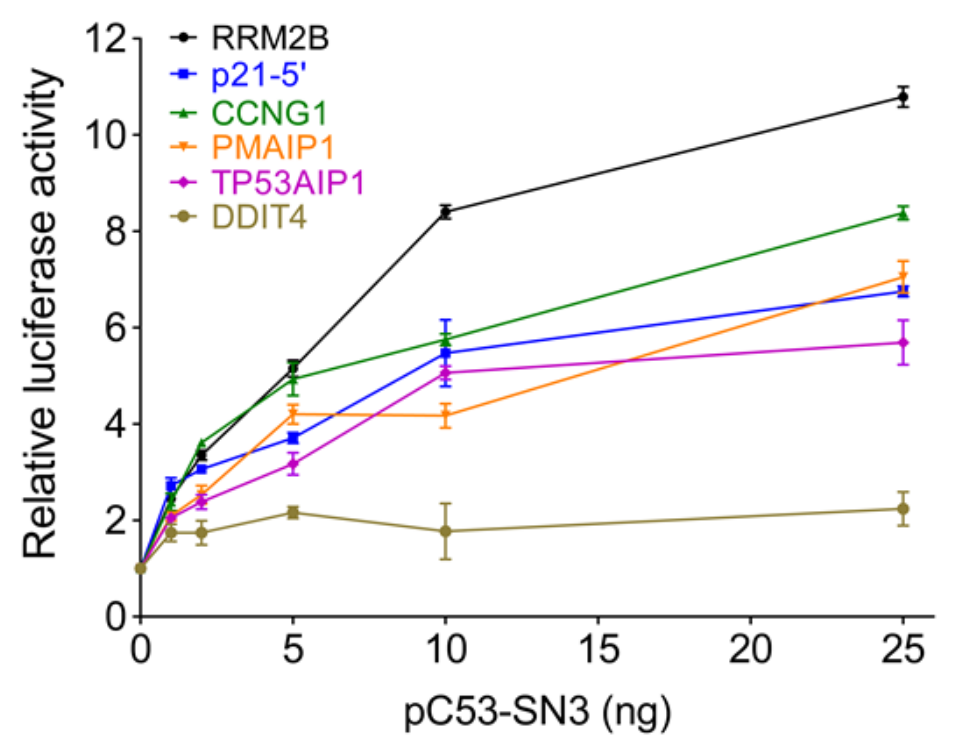

b

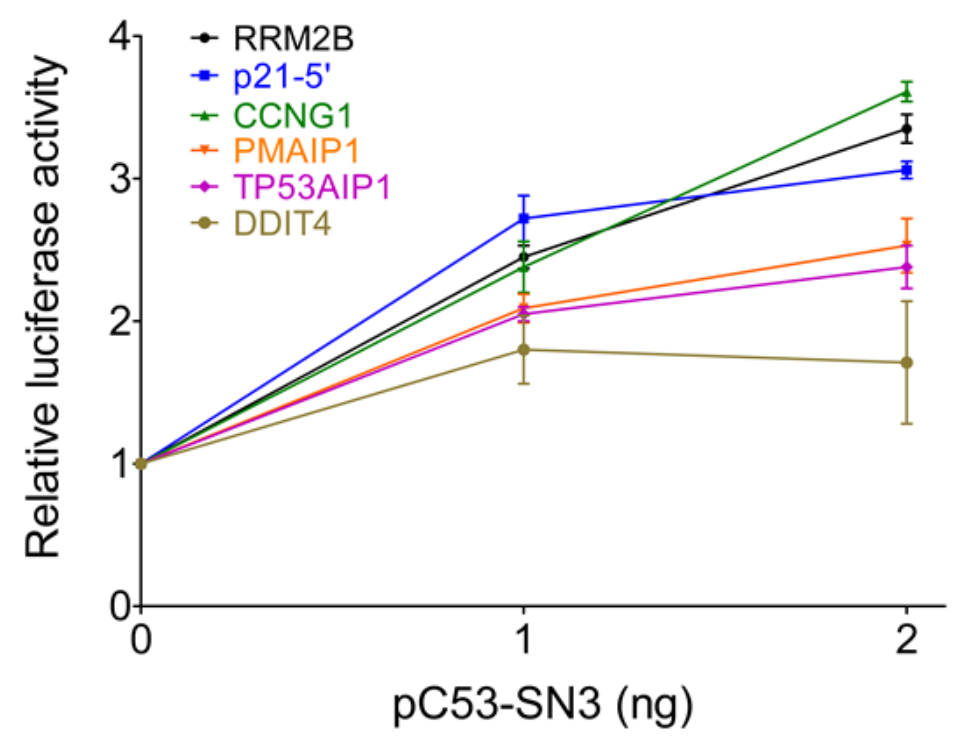

Fig. 4: Transactivation from p53 REs as a function of different p53 concentrations. Fold increase in transactivation level measured from six different p53 REs as a function of six different p53 expression levels ( $1 \mathrm{ng}, 2 \mathrm{ng}, 5 \mathrm{ng}, 10 \mathrm{ng}, 25 \mathrm{ng}$, and $75 \mathrm{ng}$ pC53-SN3 plasmid in the transfection). Luminescence was measured $48 \mathrm{~h}$ post transfection. Luminescence values were normalized to the transfection efficiency of co-transfected, constitutively expressed Gaussia luciferase. Results were normalized first to the empty cLuc vector and then to results obtained without p53. Error bars represent mean \pm SD of four to six independent experiments, each containing 2 technical replicas. (A) shows the range of p53 levels from 1 to $25 \mathrm{ng}$, (B) is an inset focusing on the 1-2 ng levels. See Figure S4 for the whole range of p53 levels, including $75 \mathrm{ng}$. 


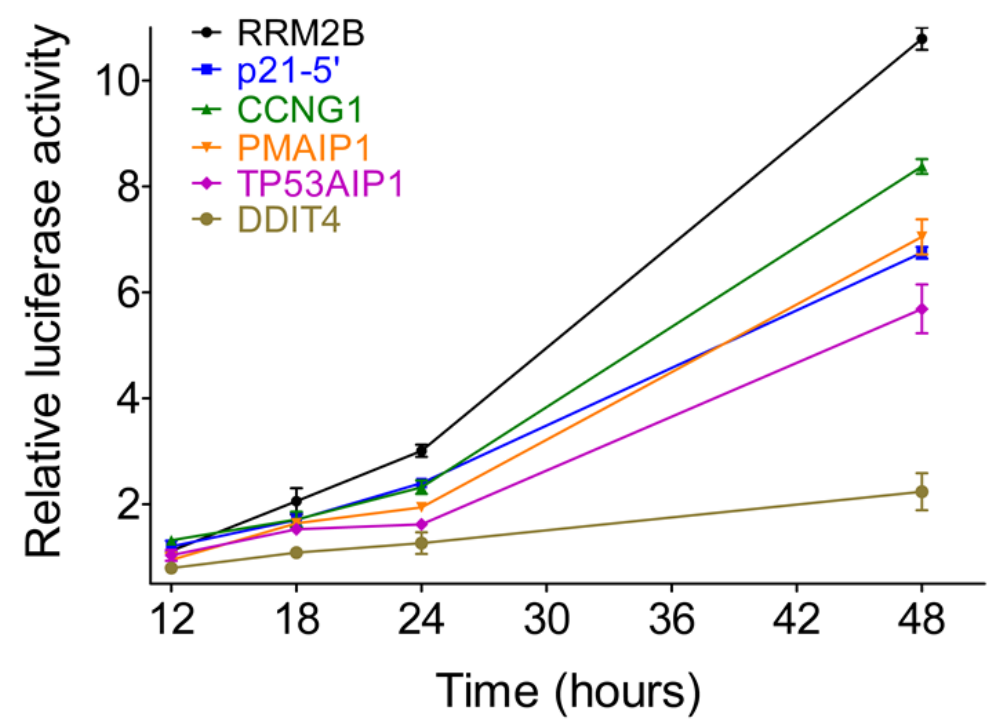

Fig. 5: Transactivation from $533 \mathrm{REs}$ as a function of time. Fold increase in transactivation level measured from six different p53 REs as a function of four time points $(12 \mathrm{~h}, 18 \mathrm{~h}, 24 \mathrm{~h}$, and $48 \mathrm{~h}$ post transfection), using $25 \mathrm{ng}$ pC53-SN3 plasmid. Error bars represent mean \pm SD of four independent experiments, each containing 2 technical replicas. Luminescence analysis details are as in Fig. 4. 
a

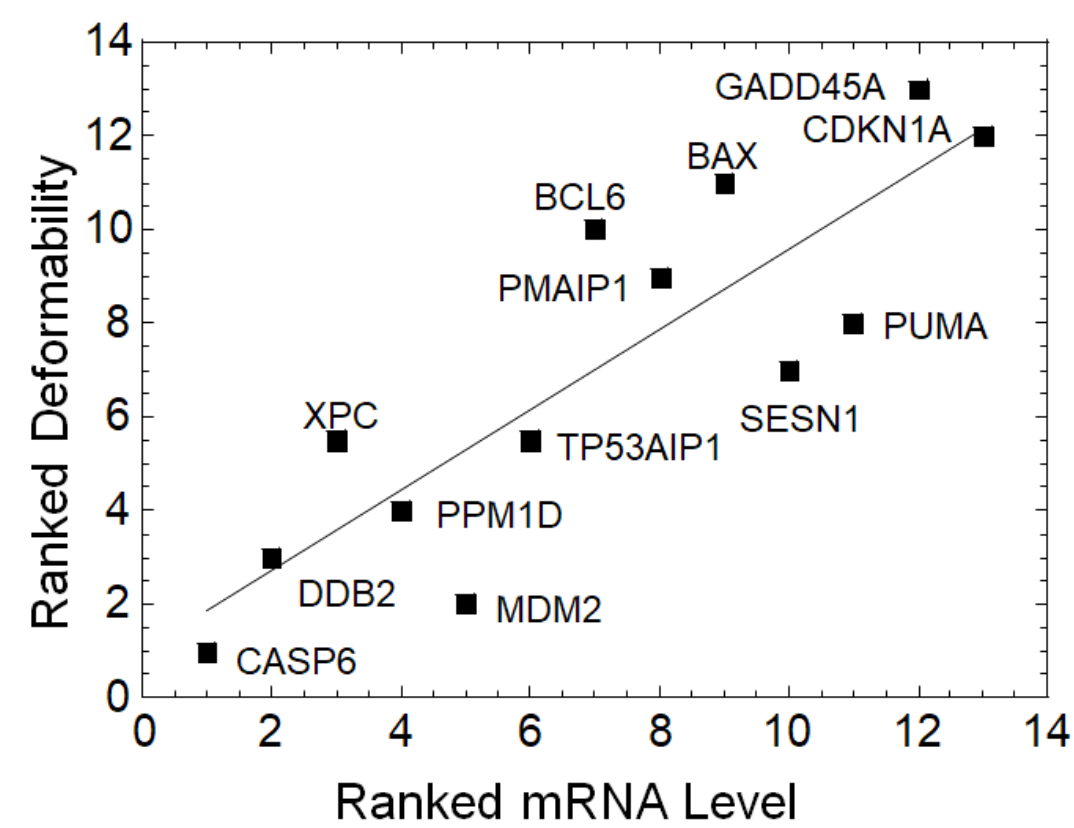

b

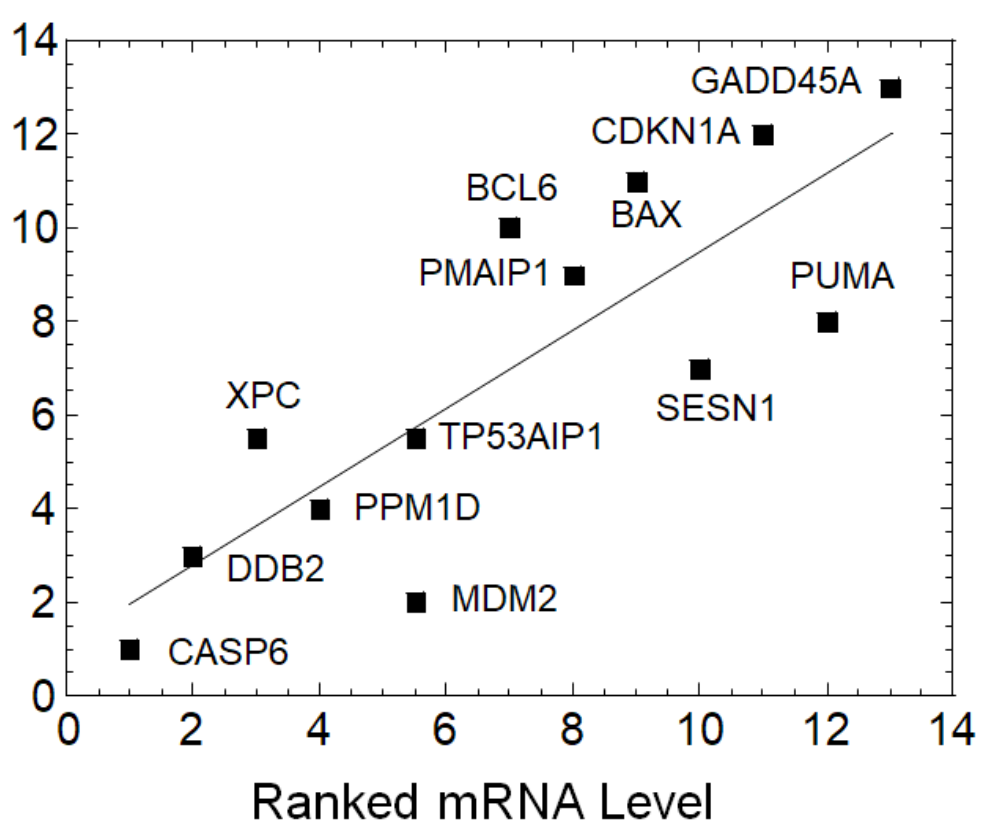

Fig. 6: mRNA level from endogenous p53-dependent genes is correlated with the calculated deformability of their REs. The mRNA level data is from Purvis et al. ${ }^{40}$, and is (A) the pulsatile expression $\left(\rho=0.88, P=8.4 \times 10^{-5}\right)$ or $(\mathrm{B})$ the sustained pattern $\left(\rho=0.85, P=2.6 \times 10^{-4}\right), 1 \mathrm{~h} \mathrm{post} \mathrm{stress}$ induction. See text for details. 
Table 1: p53 REs deformability averaged per functional outcome category

\begin{tabular}{|c|c|c|c|}
\hline Functional outcome ${ }^{\text {a }}$ & $\mathrm{V}(\mathrm{B})$ group average $\left({ }^{\left.\circ \mathbf{3}^{\circ} \AA^{3}\right)}\right.$ & Functional outcome & $\mathrm{V}(\mathrm{B})$ group average $\left({ }^{03} \AA^{3}\right)$ \\
\hline Adaptive immunity (4) & $2.08 \pm 0.03$ & Energy Metabolism (12) & $2.48 \pm 0.04$ \\
\hline Angiogenesis (8) & $2.36 \pm 0.06$ & Extrinsic apoptosis (10) & $2.28 \pm 0.03$ \\
\hline Anti-metastasis (5) & $2.34 \pm 0.05$ & Fertility \& implantation (5) & $2.33 \pm 0.09$ \\
\hline Autophagy (13) & $2.37 \pm 0.08$ & Innate immunity (20) & $2.49 \pm 0.05$ \\
\hline Cell adhesion (22) & $2.13 \pm 0.03$ & Intrinsic apoptosis (13) & $1.96 \pm 0.03$ \\
\hline Cell cycle arrest (9) & $2.36 \pm 0.03$ & Ion homeostasis (5) & $2.27 \pm 0.04$ \\
\hline Cell division (6) & $2.21 \pm 0.05$ & Membrane trafficking (9) & $2.25 \pm 0.04$ \\
\hline Cell migration (6) & $2.10 \pm 0.07$ & Oxidative stress (10) & $2.13 \pm 0.07$ \\
\hline Cytoskeleton (17) & $2.07 \pm 0.04$ & p53 negative regulation (8) & $1.96 \pm 0.06$ \\
\hline Development (15) & $2.54 \pm 0.03$ & p53 positive regulation (8) & $2.34 \pm 0.04$ \\
\hline Differentiation (18) & $2.23 \pm 0.03$ & Survival (11) & $2.19 \pm 0.04$ \\
\hline DNA damage response (10) & $2.48 \pm 0.03$ & Transcription regulation (4) & $1.85 \pm 0.05$ \\
\hline DNA repair $(9)$ & $2.23 \pm 0.06$ & Translation regulation (5) & $2.18 \pm 0.11$ \\
\hline
\end{tabular}

${ }^{a}$ Number in parenthesis denotes number of genes in the functional outcome category 
Table 2: Best-fit parameters from cyclization kinetics measurements for the sequences studied here

\begin{tabular}{|l|c|c|c|c|c|c|}
\hline Functional outcome & Gene symbol & Sequence & Bend angle $\left(^{\circ}\right)$ & $\begin{array}{c}\text { Twist angle }\left(^{\circ}\right) \\
\mathrm{b}, \mathrm{b}\end{array}$ & $\begin{array}{c}\text { Roll and Tilt } \\
\text { fluctuation }\left(^{\circ}\right) \\
\mathrm{b}, \mathrm{c}\end{array}$ & $\begin{array}{c}\text { Twist } \\
\text { fluctuation }\left({ }^{\circ}\right) \\
\mathrm{b}, \mathrm{d}\end{array}$ \\
\hline $\begin{array}{l}\text { DNA damage response } \\
\text { \& DNA Repair }\end{array}$ & RRM2B & TGACATGCCCAGGCATGTCT & $-1.24(0.11)$ & $34.16(0.11)$ & $4.31(0.12)$ & $5.60(0.12)$ \\
\hline Cell cycle & p21-5 & GAACATGTCCCAACATGTTG & $-0.06(0.08)$ & $34.04(0.09)$ & $4.45(0.08)$ & $5.34(0.08)$ \\
\hline Cell cycle & CCNG1 & GCACAAGCCCAGGCTAGTCC & $-0.72(0.09)$ & $34.52(0.08)$ & $4.54(0.09)$ & $5.77(0.09)$ \\
\hline Apoptosis & PMAIP1 & GAGCGTGTCCGGGCAGGTCG & $-1.24(0.15)$ & $34.29(0.15)$ & $4.50(0.14)$ & $5.13(0.15)$ \\
\hline Apoptosis & TP53AIP1 & TCTCTTGCCCGGGCTTGTCG & $-1.02(0.11)$ & $33.96(0.11)$ & $4.20(0.12)$ & $4.91(0.10)$ \\
\hline $\begin{array}{l}\text { mTOR inhibition \& } \\
\text { p53 regulation }\end{array}$ & DDIT4 & AAACAAGTCTTTCCTTGATC & $0.15(0.13)$ & $34.17(012)$ & $4.45(0.12)$ & $4.67(0.13)$ \\
\hline $\begin{array}{l}\text { Range of values } \\
\text { observed }\end{array}$ & & & -10.0 to 2.4 & 33.96 to 34.85 & 4.20 to 6.40 & 3.9 to 5.80 \\
\hline
\end{tabular}

${ }^{a}$ Bending is by roll and its center is located at the fourth step of all sequences, i.e. at the C-A, C-G or C-T step.

${ }^{\mathrm{b}}$ Numbers in parenthesis are the simulation errors, calculated as described in ref. ${ }^{58}$.

${ }^{\mathrm{c}}$ Roll and tilt fluctuations are the thermal fluctuations of the roll and tilt angle between adjacent base pairs ${ }^{57,58}$.

${ }^{\mathrm{d}}$ Twist fluctuations are the thermal fluctuations of the twist angle between adjacent bases ${ }^{57,58}$.

${ }^{\mathrm{e}}$ These values denote the range of observed values for these parameters in other DNA sequences, determined by the same method and the same constructs used here ${ }^{18,25,34,59}$. 